\title{
Report of the Second International Symposium on Molecular Genetics of Diabetes Mellitus"
}

\section{The meeting was held in Greifswald, FRG, 23-25 April 1990}

\author{
R. Walther and H. Zühlke \\ Institute of Biochemistry, University of Greifswald, Greifswald, FRG
}

Scientists from nine countries took part and discussed experimental results and future prospects of the following topics: (1) Hormone genes, their structure and control of expression; (2) DNA-polymorphisms and diabetes mellitus.

Introducing the first topic J. Philippe (Geneva, Switzerland) gave an overview on the genetic structure of polypeptide hormones and the potential sites involved in the control of their expression, particularly at the transcriptional level.

Experimental results demonstrating positive as well as negative regulation of the human insulin gene by multiple factors acting within its 5'-flanking regulatory sequence were presented by K. Docherty (Birmingham, UK) who also further reported on the first characterisations of a beta-cell-specific transacting factor.

A report was given by J. Hoiriis Nielsen (Gentofte, Denmark) on the expression of the growth hormone receptor gene in insulin producing cells and the stimulatory effects of the hormone on beta-cell proliferation. E.Douglas-Petersen (Gentofte, Denmark) reported on the effects of serum and growth hormone on the expression of the transcriptional factors $\mathrm{c}$-fos and c-jun in insulin producing cells. N. Welsh (Uppsala, Sweden) dealt with the role of GTP-binding proteins in the regulation of insulin biosyntheses and islet growth. For this purpose islets were transfected with respective genes using liposomes as gene carrier. R. Salazar (Greifswald, FRG) presented results indicating regulatory effects of opioid peptides on insulin gene expression. N.Blume (Gentofte, Denmark) reported on specific Cpeptide antisera which are useful in analysing the differential expression of rat and mouse insulin-I and-II genes. H.Zühlke (Greifswald, FRG) demonstrated evidence of the expression of the proopiomelanocortin gene in islets, whereby the level of expression was influenced by changes in glucose concentrations as well as by cAMP and glucocorticoids. V.N. Chesnokov (Novosibirsk, USSR) reported on hydrocortisone regulation and tissue-specific expression of the tyrosine aminotransferase gene.

\footnotetext{
* The meeting was organized by the Institute of Biochemistry of the Ernst-Moritz-Arndt-University and supported by Bayer AG, Berlin-Chemie, Novo-Nordisk, Biochemical Society of the FRG and Society of Endocrinology of the FRG
}

In his paper on advances in measurement of reporter gene A. Berthold (Wildbach, FRG) compared the chloramphenicol acetyltransferase assay with the firefly luciferase assay favouring the latter because of its higher sensitivity and less time consuming procedure.

Introducing the second topic R. Brandt (Greifswald, FRG) presented results from studies on HLA-DQ $\beta$ restriction fragment length polymorphisms (RFLP) demonstrating an association of certain fragments and fragment combinations with Type 1 (insulin-dependent) diabetes. This was supported by S. Conrad (UIm, FRG) analysing HLA-DQA1 and HLA-DQB1 using specific oligonucleotide probes and sequencing. Regarding HLA-DQA1 alleles there were no significant differences between healthy and diabetic haplotypes. With respect to the HLA-DQB1 analysis it was concluded that in Caucasian subjects aspartic acid at position 57 protects against the development of Type 1 diabetes whereas non-Asp 57 haplotypes confer suspectibility. Concerning the same HLA-molecules J.S. Petersen (Gentofte, Denmark) presented results obtained with epitope specific antibodies discriminating specific cell surface molecules differing in only one amino acid. The first results in the characterisation of HLA-DQ alpha gene alleles using polymerase chain reaction were demonstrated by V. V. Nosikov (Moscow, USSR). Investigating the Bgl II RFLP of the insulin receptor gene S. Menzel (Greifswald, FRG) did not detect differences in the allelic distribution between Type 2 (non-insulin-dependent) diabetic patients and healthy control subjects.

Estimating the complement (C 3) phenotype and allelic frequencies in diabetic patients with coronary heart disease and arterial hypertension respectively with healthy control subjects $S$. Krantz (Greifswald, FRG) had obtained no evidence that genes linked to C 3 confer susceptibility to any disease studied.

Continuing the first topic, O. Madsen (Gentofte, Denmark) reported on the development of a pluripotent transformed rat islet cell line losing its beta-cell specifity under certain conditions probably by the omission of signals responsible for maintaining the beta-cell phenotype. It was shown that putative transacting factors controlling insulin gene expression have a higher affinity to enhancer-elements of the endogenous rat gene in comparison to transfected human genes.

A.R.Clark (Birmingham, UK) described in his paper a betacell specific negative regulatory element (NRE), located in the $5^{\prime}$ flanking sequence of the human insulin gene which may play a 
physiological role in down-regulation of the human insulin gene expression.

Characterization of insulin gene binding proteins were also the subject of reports given by K.Lund (Gentofte, Denmark) and H.V.Laursen (Gentofte, Denmark). M.C.U.Hammond-Kosack (Birmingham, UK) reported on hypersensitive sites of the human insulin gene treated with DNA-structural probes bromacetaldehyde and nuclease $S 1$.

I.Leibiger (Greifswald, FRG) presented a model of liposomemediated gene transfer into rat liver cells in vivo. Using this system the liposome-mediated transfer of an insulin gene would provide the possibility of studying the insulin gene expression in liver cells maintaining their physiological surroundings. K. Keller (Berlin, FRG) used Xenopus oocytes to study the expression of different mammalian factilitative glucose transporters. R. Walther (Greifswald, FRG) discussed the use of transgenic animals in diabetes research.
A.K. Kleinschmidt (Ulm, FRG) reported on comparative studies of signal peptides in Apis mellifera and Arabidopsis thaliana. In a clinically oriented paper S. Tanneberger (Berlin-Buch, FRG) presented case reports of patients who developed diabetes during therapeutic treatment for mammary carcinoma.

The proceedings of the meeting will be published in Biomedica Biochimica Acta.

Prof. Dr. H. Zühlke

Institute of Biochemistry

University of Greifswald

Fleischmannstraße

O-2200 Greifswald

FRG 